\title{
Notes on four new synonyms of the genus Dioscorea Plum. ex L.
}

\author{
Akramul Hoque, Pakshirajan Lakshminarasimhan ${ }^{1}$ and Debabrata Maity ${ }^{2}$ \\ Taxonomy and Biosystematics Laboratory, Department of Botany, University of Calcutta, 35 Ballygunge \\ Circular Road, Kolkata 700019, West Bengal, India \\ ${ }^{1}$ Botanical Survey of India, Western Regional Centre, 7 Koregaon Road, Pune 411001, Maharashtra, India \\ ${ }^{2}$ Author for correspondence, e-mail: debmaity@yahoo.com
}

[Received 12.05.2019; Revised 22.06.2019; Accepted 25.06.2019; Published 30.06.2019]

\begin{abstract}
Four names, viz. Dioscorea bulbifera L. var. simbha Prain \& Burkill, D. bulbifera L. var. suavia Prain \& Burkill, D. oppositifolia L. var. dukhunensis Prain \& Burkill and D. pentaphylla L. var. kussok Prain \& Burkill are treated as synonyms under the typical species Dioscorea bulbifera L., D. oppositifolia L. and D. pentaphylla L.
\end{abstract}

Key words: Dioscorea, distribution, syn. nov., type.

\section{INTRODUCTION}

The genus Dioscorea Plum. ex L. is distributed in the tropical and subtropical regions of both old and new world with its 630 species (Mabberley 2017). In India, the genus is represented by 36 species and 23 varieties and a majority of the members are distributed in the Himalayan region (Karthikeyan et al. 1989; Hoque \& Mukherjee 2002; Hoque et al. 2017,2018 b). During the revisionary study of the family Dioscoreaceae in India Hoque et al. $(2017,2018 \mathrm{a})$ have added two species to the flora of India: Dioscorea polystachya Turcz. as new record for the country and Dioscorea longipedicellata Hoque, Lakshmin. $\&$ D. Maity as new to science. As a part of this revisionary work after rigorous study four varietal names, viz. Dioscorea bulbifera L. var. simbha Prain \& Burkill, D. bulbifera L. var. suavior Prain \& Burkill, D. oppositifolia L. var. dukhunensis Prain \& Burkill and D. pentaphylla $\mathrm{L}$. var. kussok Prain \& Burkill have been considered here as the new synonyms under respective three species, viz. Dioscorea bulbifera, D. oppositifolia and D. pentaphylla. Though the World Checklist (https://wcsp.science.kew.org/qsearch.do) mentioned the three names, viz. D. bulbifera L. var. suavia Prain \& Burkill, D. oppositifolia L. var. dukhunensis Prain \& Burkill and D. pentaphylla L. var. kussok Prain \& Burkill under synonymy of typical plants, however, do not provide any explanatory notes. Thus, explanatory notes for each taxon are provided for proper justification.

\section{MATERIALS AND METHODS}

Many field trips have been conducted in different phytogeographical regions of the country and rigorous collections have also been made from these localities. Specimens are studied carefully in the field as well as in the laboratory to note the variability. Herbarium specimens deposited at CAL, MH, BSIS, BSI, ARUN, BSHC and CUH were examined carefully. High resolution digital images deposited at K, BM, E, P, etc. have also been analyzed. Extensive 
literature survey including protologue has also been done to justify the observation. All type specimens under these names deposited at CAL were also critically examined.

\section{RESULT}

Dioscorea bulbifera L., Sp. Pl. ed. 1: 1033. 1753; Thwaites, Enum. Pl.. Zeyl.: 326. 1864; Wight, Icon. Pl. Ind. Orient. 3(2): t. 878. 1844-45; Hook. f., Fl. Brit. India. 6: 296. 1892; Prain, Bengal Pl. 2: 1066. 1903; T. Cooke, Fl. Bombay. 3 : 265. 1908; Merr., Interpr. Herb. Amboin.:: 146. 1917; Duthie, Fl. Gangetic Plain 3(2):254.1920; Kunth in Engl., Pflanzenr. 87 $($ IV-43) : 88. 1924 p.p. (excl. D. eburina Lour.); C.E.C. Fisch. in Gamble, Fl. Madras 3 : 1055. 1928; Haines, Bot. Bihar Orissa 3: 1172. 1924; Prain \& Burkill in Ann. Roy. Bot. Gard. Calcutta 14(1) : 111, t. 49. 1936; Burkill in Steenis, Fl. Males., Ser. 1, Spermat. $4: 311$. 1951; H. Ohashi in H. Hara, Fl. E. Himalaya: 419. 1966; Siddiqi in Nasir et Ali, Fl. W. Pakistan 53: 5. 1973; Gandhi in C.J. Saldanha \& Nicolson, Fl. Hassan Distr.: 808. 1976; Babu, Herb. Fl. Dehra Dun: 515. 1977; H. Hara et al., Enum. Fl. Pl. Nepal 1: 67. 1978; K.M. Matthew et Britto in K.M. Matthew, Fl. Tamilnadu Carnatic 3: 1633. 1983; Deb, F1.Tripura State 2: 419. 1983; Nicolson et al. Interpret. Van Rheede's Hort. Malab.: 290. 1988; Karthik. et al., Fl. Ind. Enum. Monocot. : 73. 1989; Noltie, Fl. Bhutan 3(1): 9. 1994; Jayas. in Dassan. Revis. Handb. Fl. Ceylon 9: 52. 1995.

D. bulbifera L. var. simbha Prain \&Burkill, J. Proc. Asiat. Soc. Bengal 10(1) : 26. 1914; Prain \& Burkill, Ann. Roy. Bot. Gard. Calcutta 14(1): 117, t. 49(4 \& 5). 1936; Noltie, Fl. Bhutan 3(1): 9.1994, syn. nov.

Lectotype: India, West Bengal, Darjeeling, Mungpu, 2800 ft, 31.7.1903, A.C. Hartless 1, acc. no. 475530 CAL! (designated by Hoque et al., 2018); Syntypes: Meghalaya, Cherrapunjee, 11.8.1912, R.K. Das 35633 (CAL0000026164); Sikkim Himalaya, Pashok, Oct. 1903, J.L. Lister s.n., acc. no. 475525 CAL!; West Bengal, Eastern Himalaya, Sureil, 5000 ft, 21.9.1912, P.T. Russell 27, CAL!; Mungpoo, 2500 ft, 21.8.1912, P.T. Russell 21, CAL!

D. bulbifera L. var. suavia Prain \& Burkill, J. Proc. Asiat. Soc. Bengal 10(1): 26. 1914; Duthie, Fl. Gangetic Plain 3(2):254.1920; Prain \& Burkill, Ann. Roy. Bot. Gard. Calcutta 14(1) :117, t 51(4, 5 \& 6). 1936; Burkill in Steenis, Fl. Males., Ser. 1, Spermat. 4(1) :312. 1951; Karthik. et al., Fl. Ind. Enum. Monocot. : 73. 1989; Noltie, Fl. Bhutan 3(1): 10. 1994, syn. nov.

Lectotype: West Bengal, Darjeeling, Tista, 4 Oct. 1912, A.T. Gage 38773, acc. no. 476862 CAL! (designated by Hoque et al., 2018); Syntypes: Madhya Pradesh, Bhopal, Narashingarh, 18.7.1904, Herb. R.E.P. KalkapershadTewaris.n., acc. no. 477020 CAL!; Maharashtra, Thana, Kalyan, 22 Sept. 1910, D. Hooper 34638, acc. no. 476944 CAL!.

Note: The variety $D$. bulbifera L. var. simbha Prain \& Burkill was established by the authors based on size and taste of tuber and bulbils, brighter lower leaf surface and larger flowers. During the revisionary study of the genus Dioscorea a considerable number of specimens of $D$. bulbifera, both field and laboratory, have been studied including the specimens of $D$. bulbifera var. simbha. Importantly, the type specimens of $D$. bulbifera var. simbha deposited at CAL were also critically examined. The studied specimens were collected from different phytogeographical regions with different climatic conditions throughout its distributional range in the country starting from Jammu \& Kashmir to Andaman \& Nicobar Islands through Himachal Pradesh, Punjab, Uttar Pradesh, Uttarakhand, Sikkim, West Bengal, Assam, Meghalaya, Arunachal Pradesh, Nagaland, Mizoram, Manipur, Tripura, Bihar, Jharkhand Odisha, Andhra Pradesh, Telengana, Tamil Nadu, Kerala, Karnataka, Goa, 
Maharashtra, Madhya Pradesh, Chhattisgarh, Gujarat and Rajasthan. Moreover, D. bulbifera is a widely distributed species from Africa to Australia through India, Nepal, Bhutan, SouthWest China, Japan, Bangladesh, Myanmar, Malaysia, Thailand, Laos, Indonesia, Philippines, Sri Lanka, New Guinea and Pacific Islands and shows vast morphoplasticity in most of its vegetative characters. Moreover, some reproductive characters, viz. size of flowers and capsule is also varies considerably across the populations as well as within the same individual. After critical study it has been found that all the characters used by Prain and Burkill (1914) to diagnose their variety are fall under range of variations without showing any consistency and correlation. The size of leaf lamina is extremely variable $(10.5-33 \times 4.7-27.5 \mathrm{~cm})$ between the populations as well as even in an individual. The length of flowers varies from $1.5 \mathrm{~mm}$ to $6 \mathrm{~mm}$ even in the same material as evident from the studied specimens. Importantly the taste of tuber and bulbil could not be considered as a diagnostic tool for a taxon. Therefore the var. simbha is presenty considered as a synonym under the typical species Dioscorea bulbifera $\mathrm{L}$.

Prain and Burkill (1914) established D. bulbifera var. suavior Prain \& Burkill based on the specimens collected from Darjeeling (West Bengal), Bhopal (Madhya Pradesh) and Thana (Maharashtra). They separated their new taxon from the typical one by the characters, viz. long, large tuber having hairy roots and more or less with acrid taste and warty bulbils. After critical examination of huge number of specimens of both $D$. bulbifera var. bulbifera and D. bulbifera var. suavior including the type specimens of the later taxon deposited at CAL it has been noticed that both the characters used by the authors are much variable even in a population. Moreover, the reproductive characters, viz. size of flowers and capsules are variable in the same individual as well as within the population. The tuber size is extremely variable $(3.5-10 \times 7-20 \mathrm{~cm})$ in the individuals of a population. The rootlets on the surface of tuber also vary considerably. The size of bulbil and the warted surface of bulbils are also variable in the same material. Importantly the taste of tuber and bulbil could not be considered as a criterion to establish a new taxon. Noltie (1994) mentioned these varietal names following Prain and Burkill (1914). However, he admitted the difficulties regarding separation of all varieties recognized by Prain and Burkill (1914) under D. bulbifera in herbarium specimens and stated that "(not distinguishable from herbarium specimens and not separated above)"(p.9). He described four varieties including these two under the typical plant.

Therefore, D. bulbifera var. suavia is presently considered as a synonym under the typical species D. bulbifera L.

Distribution: INDIA : Almost in all the states, growing from sea level to an altitude of 1829 m; PAKISTHAN; NEPAL; BHUTAN; BANGLADESH; MYANMAR; SRI LANKA; SOUTH-WEST CHINA; JAPAN; MALAYSIA; THAILAND; LAOS; INDONESIA; PHILIPPINES; NEW GUINEA; AUSTRALIA; PACIFIC ISLANDS; AFRICA. Cultivated in some parts of India for its starchy tubers and bulbils.

Flowering: July to September; Fruiting: September to December.

\section{Specimens examined:}

JAMMU \& KASHMIR : P.K. Hajra 82545 (BSD); HIMACHAL PRADESH: Gamble 4928D; Grant s.n.; Clarke 24109C; Meebold 620; Lace 799; Gamble 4926C; King 830; King 622; Kalkaprasad 28763; Johnson 15; Thomson s.n.; Clarke 132 (all at CAL); Vohra 54239 (BSD); Lace 2066 (BSIS); PUNJAB : Misra 41739 (CAL); UTTAR PRADESH: Mackinnon sn.; Kanjilal 1174 (all at ASSAM); Mackinnon sn.; King sn.; Gamble 27129 ; King s.n. ; King s.n. ; Duthie 2074 ; Rau 6319 ; Herb. R.E.P. 20973 ; 
Panigrahi 2221 ; Herb. R.E.P. 18083, Cult. H.B.C. 21054 ; Burkill et Prain s.n.; Khanna \& R. Saran 39196 ; Khanna \& R. Saran 39186 ; Panigrahi 12424 ; Arora 49974 ; Malhotra 504221 ; Mitra, Akramul Hoque \& Party 1 ; 2 ; Bhattacharyya 33655 ; Prasad 333 ; Herb. R.E.P. 19020, Cult H.B.C. 21066 ; Strachey \& Winterbottom 2 ; Akramul Hoque 139 ; 140; Kalkaprasad 34803; Panigrahi 3652; King s.n.; Khanna \& Saran 38654; Akramul Hoque 22; 23 (all at CAL); Arora 49973; Arora 49599; 49600; Janardhanan 50197 (all at CAL \& BSD); Rau 6318; 6119; Goel 64593; 64600; Ahsan \& R.R. Srivastava 68404; Karki 68634; Panigrahi 65284; Arora 45465; Pant \& Naithani 39560; Balodi 75066; Vyas s.n.; Rao 1013; Karki 82067; Misra 37931; Malhotra 50637; Babu 33252; Malhotra 51013; Singh 219; Bhattacharyya 17670; Malhotra 50647; Arora 37895; Malhotra 22762; Malhotra 22895; Misra 38228; Bhattacharyya 16092; Malhotra 33939; Prasad \& Singh 86745; Rau 10405; Saran 8828; Uniyal 72756 (all at BSD); Maheshwaram s.n. (MH); SIKKIM: Akramul Hoque 20575; 20576; Akramul Hoque 20570; Rao 1111; King s.n.; Hooker s.n.; King s.n.; Akramul Hoque 20571; Hajra 263; Sengupta 305; Majumdar \& Banerjee 629; Prain's Collector s.n.; Prain s.n.; Lister s.n.; Biswas 9756; Edgeworth s.n.; King 5C; 5D; Treutler s.n. (all at CAL); Hooker s.n. (MH); Anderson 1302 (CAL, MH); Hajra 557 (BSHC, CAL); Raju 3917; Varma 6798; 6800; Raju \& Singh 5649; Russell 11; Pradhan 15061; Mandal 11106 (all at BSHC); WEST BENGAL: Sikdar 677; Malick 462; Biswas 4685; Malick 435; Narayanaswami \& Party 3072; Malick 78; Russell 21; Helfer 128; Johnson s.n.; Russell 27; Ripley 60; Haines 485; Gamble 9797; Clarke 12088; Narayanaswami 1132; Sanyal 757; Bennet 945; Mukherjee 18755; Sanyal 188; Mukherjee 4557; Janaki Ammal 30/56; R.S.R. 1567; Hosein 1902; Sen 853; Chatterjee 19; Burkill \& Prain S.n.; Gage 34228; Herb. R.E.P. 18563, Cult H.B.C. 21063; Herb. R.E.P. 18564, Cult H.B.C. 21043; Akramul Hoque 18071; Herb. R.E.P. 19131, Cult H.B.C. 21052; Das 35402; 30749; Burkill 32358; Cousins 48; Akramul Hoque 18703; 18704; Herb. R.E.P. 13815, Burkill \& Prain s.n.; Herb. R.E.P. 13915; Watt, Herb. R.E.P. 13915, Cult H.B.C. s.n.; Herb. R.E.P. s.n.; Herb. R.E.P. 11693, Burkill \& Prain s.n.; Das 35461; 35567; Russell 23; Hartless 2; Raghavan 1572; Raghavan 1131/59; Bennet 785; Bennet 1000; Sanyal 167; Russell 13; Watt, Herb. R.E.P. 11807, Cult. H..B.C., Prain's Collector s.n.; Herb. R.E.P. 13908, Cult. H.B.C. s.n. , Burkill \& Prain s.n.; Safui \& party 10379; Sikdar 644; Herb. R.E.P. 19234, Cult. H.B.C. 21045; Lister s.n.; Sengupta 130; Chatterjee 21; Lister s.n.; Herb. R.E.P. 20057, Cult. H.B.C. 21068; Raghavan 1566; 1568; 1569; 1570; 1571; Raghavan 917; Dutta 318; Sen 742; Malick 278; Guhabakshi 1034; Malick 388; Raghavan 918; Das 34215; Cardon, Herb R.E.P. s.n.; Herb. R.E.P. 18551, Cult. H.B.C. 21060; Herb. R.E.P. 18185, Cult H.B.C. 21076; Cult. H.B.C. s.n.; Das 34752; Basak 538; Malick 432; Burkill 30723; Akramul Hoque 20510; Mann 4; Herb. R.E.P. 13950, Burkill \& Prain s.n.; Gamble 9744; Clarke 11928B; Anderson 1297; Clarke 8807; Gamble 1365D; Majhi 1690 (all at CAL); Hartless 1 (BSIS, CAL); ASSAM: Prazer s.n.; Craib 180; Burkill 32418; Burkill 32630; Munro s.n.; Simons s.n.; Anderson 1302 (all at CAL); Bal 326 (CAL, BSIS); Bor 15210; Panigrahi 11294; Rao 42475; Deka 18142; De 18278; Math 13365; De 18206; Kanjilal s.n. (all at ASSAM); MEGHALAYA : Balakrishnan 42773; Deka 19640; Deka s.n.; Sharma 13575; Deka 22519; Deb 29159; Balakrishnan 47043; Naik s.n. (all at ASSAM); Hooker \& Thomson s.n. (MH); Akramul Hoque 20536; 20537; 20538; 20539; 20540; 20562; 20563; 20572; 20558; 20552; 20554; Hooker \& Thomson s.n.; Deka s.n.; Kapoor \& Party 75688; Carter 1655; 846; Burkill \& Banerjee 268; 35116; 35118; Das 35633; 35631; Hooker s.n.; Hajra 20501; Griffith 50 (all at CAL); Panigrahi 3634 (CAL, ASSAM); ARUNACHAL PRADESH: Rao 10254; Joseph s.n.; Deb 26199 (ASSAM); Pramanik 5124; 5268; 409; 6477; Haridasan 301; 2654; 4499; 3378; 4762; 3796 (all at ARUN); Akramul Hoque 205267; 20512; 20512; 20535; 20531; 20516; Bhoumik \& Pathak 2086; 20518; 20524; 20525; 20526; Rao 47942; Panigrahi 14868; 16918; Deb 26199 (all at CAL); Panigrahi 19341; Panigrahi 14778; Rao 19974; Deb 26650; Panigrahi 14580; Deb 26091; Josheph 39792 (all at CAL \& ASSAM); NAGALAND : Prain s.n.; Clarke 41377A (all at CAL); MIZORAM: Prazer s.n.; Parry 
s.n. (All at CAL); Dutta 33230 (ASSAM); MANIPUR: Malick 849 (BSIS); TRIPURA: Deb 1055 ; 1003; 2580; Bagapasha, Deb 1076 (all at CAL); Rao 8831 (CAL, ASSAM); BIHAR: Clarke 20925; Prain s.n.; Campbell 7571; Chandra 913; S.K.B. \& B.N.C. 8; Burkill 27360; Kanodia 1330; Banerjee 139; 577; 406; Shetty 263; 337; 200; Burkill \& Prain s.n.; Herb. R.E.P. 13895, Burkill \& Prain s.n.; Mukherjee 1914; Watt, Herb. R.E.P. 13813, Prain's Collector s.n.; Cult. H.B.C. 21079; Herb. R.E.P. 20857, Prain \& Burkill s.n.; Prain s.n.; Cardon 9; 13; 15 (all at CAL); Haines 186 (CAL, MH); ORISSA: Panigrahi 20899 (CAL, ASSAM); Narayanaswami 5791; 5950; Haines 5119 (all at MH); Herb. R.E.P. 15492, Burkill \& Prain s.n.; Herb. R.E.P. 18472, Cult. H.B.C. s.n.; Herb. R.E.P. 18100, Cult. H.B.C. 21072 ; Herb. R.E.P. 18472, Cult. H.B.C. 21062; Herb. R.E.P. 18965, Cult. H.B.C. 21053; Panda \& Das 773; Herb. R.E.P. 13920, Burkill \& Prain s.n.; Annandale 1273; Namhata 37788 ; Safui \& Party 13528; 13388; Amandale 1273; Watt. Herb. R.E.P. 13923, Gage s.n. (all at CAL); ANDHRA PRADESH: Barber 5343; Balakrishnan 10750; Sebastine 13117 (all at CAL \& MH); Watt, Herb. R.E.P. 11807 (CAL, BSIS); Ravishankar 83687; Ellis 42195; S. coll.12070, 12051, 12053; Subba Rao, 67529; 21810; 42516; 42532; 42644; 44423 (all at MH); Raju 69; Schatler 198; Raju 1084; Narayanaswami 535; Raju 410; Balakrishnan 977; Foulkes 88; Mohan 502; Burkill \& Prain s.n.; Herb. R.E.P. 12686, Burkill \& Prain s.n.; Balakrisnan 850; 880; Raju 389; Ramaswami 1640; Rao 67529 (all at CAL); TAMIL NADU : Viswanathan 996; 886; Sebastine 17286; Ramamurthy 18171; Vajravelu 42896; Rathakrishan 39176; Vivekananthan 43082 (all at MH); Vivekananthan 46737 (CAL, MH); Burkill 17618 (CAL, BSIS); Herb. R.E.P. 17620, Cult. H.B.C. 21069; Herb. Kew. s.n.; Raghavan 156 (all at CAL); KERALA : Ellis 20459; Vajravelu 26001; Sebastine 22322; Barber 7144 ; Antony 683; Sharma 42480; S.coll. 9235; Lauson s.n.; Sebastine 22377; Joseph 41968; Ramachandran 52239; Sebastine 25313; Ellis 25719 (all at MH); Joseph 51420; Nair 50799; Ramamurthy 74728; Mohanan 74608; Mohanan 74677; Mohanan 58314; Ansari 74348; Mohanan 65168; Mohanan 80006; Vajravelu 48898; Nair 64450; Nair 64505; Chandrabose 49023; Chandrabose 49081; Mohanan 58363 (all at CAL \& MH); Bourdillon s.n.; Thomson s.n.; Ramaswami 132; Bourdillon 1437; Herb. Wight 2822; Bourdillon 21, Cult. H.B.C. s.n.; Bourdillon s.n., Cult H.B.C. 21075; Bourdillon s.n., Cult. H.B.C. 21084; Bourdillon 24, Cult H.B.C. 21077; Ramaswami 81; Bourdillon 1266; 1416; 1265; 26567 (all at CAL); KARNATAKA : Barber 2480 (MH); Talbot 1977; Gamble 14713; Rao 94838; Rao 74928; Meebold 10077; Talbot 157 (all at CAL); GOA: Kanodia 89488; 89598; 8932; 89758 Cherian 106064;106136;106224; Ansari 93718 (all at CAL); MAHARASHTRA: Herb. R.E.P. 13855; Wood s.n.; Herb. R.E.P. 11693, Cult. H.B.C., Burkill \& Prain s.n.; Dalzell s.n.; Venkata Reddi 99215; Herb. R.E.P. 16208; 16209; Herb. R.E.P. 16209, Burkill \& Prain s.n.; Ryan Esq. 37; Hocks s.n.; Hooper 3464; 34471; 34638; Herb. R.E.P. 15772A, Burkill \& Prain s.n.; Herb. R.E.P. 16208, Burkill \& Prain s.n.; Meebold 8797; Herb. R.E.P. 16209, Burkill \& Prain s.n. (all at CAL); MADHYA PRADESH: Subramanyam 8590; Sebastine 8885; Joseph 12876; 11233 (all at MH); Jaui 5164; Panigrahi 5628; Herb. R.E.P., Kalkaprasad s.n.; Kalkaprasad s.n.; Narayanaswami 3110; King 60;122 ; Herb. R.E.P. 19454, Cult. H.B.C. 21047 ; Herb. R.E.P. s.n., Cult. H.B.C. 21051; Herb. R.E.P. 16166, Burkill \& Prain s.n.; Kalkaprasad 20214; Herb. R.E.P. 16120, Burkill \& Prain s.n.; Cult. H.B.C., Burkill \& Prain s.n.; Herb. R.E.P. 14047, Burkill \& Prain s.n.; Herb. R.E.P. 11481, Burkill \& Prain s.n.; Herb. R.E.P. 15443, Burkill \& Prain s.n.; Herb. R.E.P. 16141, Burkill \& Prain s.n.; Herb. R.E.P. 15819, Burkill \& Prain s.n.; Herb. R.E.P. 15450, Burkill \& Prain s.n.; Herb. R.E.P. 18666, Cult. H.B.C. 21057; Herb. R.E.P. 9155, Prain s.n.; Watt, Herb. R.E.P. 9155, Cult. H.B.C. s.n.; Ali Rajpur, 20.5.1897, Herb. R.E.P. 9155, Burkill et Prain s.n.; Herb. R.E.P. 11693, Burkill \& Prain s.n.; B.S.I. 5794; Panigrahi \& Singh 4247; Rao 
83910; Roy \& Kishore 44976; Herb. R.E.P. s.n., Prain's Collector s.n.; Mukherjee s.n.; Watt. Herb. R.E.P. 15450, Cult. H.B.C. s.n.; Panigrahi \& Singh 4487 (all at CAL); Joseph 14377 (CAL, MH); GUJARAT: Joshi 696 (BSIS); Saxton 1100 (CAL); RAJASTHAN: Sharma 2279; Singh \& R.P. Pandey 6947; Wadhwa 3583; Singh 5710; Singh 3095; Powlett s.n.; Singh 2993; Wadhwa 7542 (all at CAL); ANDAMAN \& NICOBAR ISLAND : King's Collector s.n.; King's Collector sn.; King's Collector s.n.; King's Collector s.n.; Helfer 5539; 554; King's Collector s.n.; King's Collector s.n.; Heinig 101H ; Prain's Collector 40; 49; Heinig s.n.; King's Collector s.n.; Sharma 4591; Basu 6626; Basu 7041; Balakrishnan 6499; Heinig 135; Heinig 627; Prain s.n.; King's Collector s.n.; King's Collector s.n.; King s.n.; King's Collector; Prain's Collector s.n.; King's Collector s.n.; Heinig s.n.; Man 64; 74; Heinig 340; Prain's Collector 310; Herb. R.E.P. 23829, Rogers s.n. (all at CAL).

Dioscorea oppositifolia L., Sp. Pl. ed.1: 1033.1753 p.p.; Willd., Sp. Pl. 4 : 791.1805 p.p.; Roxb., Hort. Bengal.: 105. 1814; Roxb., Fl. Ind. (Ed. Carey) 3:804. 1832; Wight, Icon. Pl. Ind. Orient. 3(1): 7, t. 813. 1844; Voigt, Hort. Suburb. Calcutt. (Ed. Griff.): 653. 1845; Kunth, Enum. P1.. 5 : 390, 1850 p.p.; Thwaites, Enum. Pl. Zeyl.: 326. 1864; Hook. f., Fl. Brit. India, 6: 292, 1892 p.p. (excl. D. trinervia Roxb.mss.; Wall Cat. 5104 a \& 5105e p.p.); Hook. f. in Trimen, Handb. Fl. Ceylon 4: 276. 1898; Woodrow, J. Bombay Nat. Hist. Soc. 5: 523. 1899; Prain, Bengal P1. 2 : 1278. 1903; T. Cooke, Fl. Bombay 3 : 266. 1908; Prain \& Burkill, J. Proc. Asiat. Soc. Bengal 10(1) : 30. 1914; Kunth in Engl., Pflanzenr. 87(IV-43) : 286. 1924, p.p.; Haines, Bot. Bihar Orissa 3: 1168. 1924; C.E.C Fisch. in Gamble, Fl. Madras 3 : 1056. 1928; Prain \& Burkill, Ann. Roy. Bot. Gard. Calcutta 14(2): 392. 1939, t. 139. 1938; Karthik. et al., Fl. Ind. Enum. Monocot.: 75. 1989; Jayas. in Dassan., Revis. Handb. Fl. Ceylon $9: 65.1995$.

D. oppositifolia L. var. dukhunensis Prain \& Burkill, J. Proc. Asiat. Soc. Bengal 10(1) : 30, 1914; Kunth in Engl., Pflanzenr. 87(IV-43) : 286, 1924; C.E.C. Fisch. in Gamble, Fl. Madras 3:1056, 1928; Prain \& Burkill, Ann. Roy. Bot. Gard Calcutta 14(2) : 393, 1939; Karthik. et al., Fl. Ind. Enum. Monocot.: 75. 1989; Jayas. in Dassan., Revis. Handb. Fl. Ceylon 9 : 69, 1995, syn. nov.

Lectotype: Peninsula Indiae Orientalis, Neelgherry Hill (Nilgiri Hill), July,1847, Wight 2825, K (K000098235, image!) (designated by Jayasuriya, 1995); Isolectotype: Peninsula Indiae Orientalis, Anamallay Forest, Sept. 1851, Wight 2825, acc. no. 474464, CAL (CAL0000025588!); Oothacalmundapum(Oodhagamandalam/ Ooty), December, 1847, Wight 2825 a, Upper male twig, K (K001142956, image!); Wight 2825 b, Lower female twigs, K (K001142959, image!).

Note: Dioscorea oppositifolia L. var. dukhunensis Prain \& Burkill (Prain \& Burkill, 1914) was described on the basis of leaf shape, surface and position of the inflorescence rachis, as well as the arrangement of flowers on the rachis. Jayasuriya (1995) also maintained this variety in his treatment of the species Dioscorea oppositifolia. Moreover, he also designated the lectotype of the name Dioscorea oppositifolia var. dukhunensis Prain \& Burkill. During the present revisionary study of the genus Dioscorea in India huge number of specimens of $D$. oppositifolia has been studied both in the field and the laboratory including the type specimens of D. oppositifolia var. dukhunensis deposited at CAL and in other Indian herbaria. The digital images of the specimens deposited at different world herbaria (K, E, BM, P, etc.) are also consulted for better understanding of the characters. The studied taxa have been known to be reported almost from every corner of the country starting from Rajasthan to West Bengal through Maharashtra, Goa, Karnataka, Kerala, Tamil Nadu, 
Madhya Pradesh, Andhra Pradesh, Odisha, Bihar, Andaman \& Nicobar Islands, Laksha Dweep and also from Sri-Lanka. In all the studied specimens the vegetative characters showed a vast morphoplasticity. After critical study it has been concluded that all the characters used by Prain and Burkill (1914) to diagnose their variety are fall under range of variations without showing any consistency and correlation. The shape of leaf lamina and surface are extremely variable between the populations as well as even in an individual. Laminar shape may be lanceolate, ovate, rounded, obovate to rarely ovate-cordate or rounded-cordate and laminar surface also varies from perfectly glabrous to variously hairy. Inflorescence spike always axillary, glabrous or hairy bearing both laxly or compactly arranged flowers in the same material. At present not a single character persists that could be used to separate or distinguish D. oppositifolia var. dukhunensis from typical form of the species as traditionally done by Prain and Burkill (1914), Jayasuriya (1995) and others. Therefore, D. oppositifolia var. dukhunensis Prain \& Burkill is recognized and treated here as synonym of $D$. oppositifolia L.

Flowering: July to October; Fruiting: September to January.

Distribution: Growing at the altitudes from 152 to $1829 \mathrm{~m}$. INDIA : West Bengal, Bihar, Orissa, Andhra Pradesh, Tamil Nadu, Kerala, Karnataka, Goa, Maharashtra, Madhya Pradesh, Rajasthan, Andaman \& Nicobar Islands, Laksha Dweep ; SRI-LANKA.

\section{Specimens examined:}

WEST BENGAL : Das \& Samanta 81 (N.B.U.); Sikdar 586; Mukherjee 5440 (All at CAL); BIHAR : Panigrahi 11701 (ASSAM); ORISSA : Panigrahi 8232; Namhata 2277; Safui \& Party 13387 ; 13389; Cult. H.B.C., Burkill \& Prain 11066; Cult. H.B.C. Herb. R.E.P. 18471; 18469; Cult. H.B.C., Burkill \& Prain 13924; Annandah 1331; Safui \& Party 13442; Annandale 1259; Burkill \& Prain 15821; Cult. H.B.C., Herb. R.E.P. 18539; Watt, Cult. H.B.C., Prain's Collector 9156; Watt, Cult. H.B.C. Gage 13924; Cult. H.B.C., Bourdillon 15; 17; Mukherjee 4361 (all at CAL); Panigrahi 18423 (CAL, ASSAM); Narayanaswami 5976 ; Narayanaswami 6065; Narayanaswami 5958 (all at MH); ANDHRA PRADESH : Rao 83603; Ellis 14915; Charyulu 313; Beddome s.n; Barber 5283; Beddome s.n.; Rajendraprasad 313; Venkanna 5320; Ravishankar 83689; Ellis 16926; Ellis 25408; Sebastine 6701; Jacob 17152; Balakrishnan 10968; Subba Rao 19641; Subba Rao 28077; Subba Rao 32800; Subba Rao 42526; Subba Rao 44419; Srikumar \& Rama Rao 76863 (All at MH); Narayanaswami 362; Barber s.n; Ramaswami 1370; Gamble 20956; Narayanaswami 18; Ramaswami 1577; Narayanaswami 264; Mohan 497; Wight 2825; Raju 347; Ramaswami 1646; Balakrishnan 879; Balakrishnan 1045; Balakrishnan 1118; Raju 408 (All at CAL); Subba Rao 46877; Ellis 14941; Balakrishnan 10816; Subba Rao 68654; Subba Rao 68663; Subba Rao 67550; Gamble 15093; Gamble 15179 (all at CAL \& MH); TAMIL NADU : Sebastine 844; 6250; 6980; 12949; 5439; 9541; 12940; 1387; 7021; 14405; 14405; Vajravelu 82220; 77745; 80698; 51982; Joseph 12675; 14160 ; Joseph 15186; Ramamurthy 14874; 53529; Subramanyam 1477; 1267; 1955; 8280; 824; 8082; Henry 48162; 48314; 68924; 68926 (all at CAL \& MH); Herb. Wight 1092; Ramaswami 1646; 39339; Cult. H.B.C. s.n. acc. no. 474447; Ragupathy 1012; Subramanyam 8278; Subramanyam 1267; 876; 6083; Akramul Hoque 19906; Cult. H.B.C. s.n. acc. no. 474448; Cult. H.B.C., R.E.P. 18956; Bourdillon 6; Cult. H.B.C., R.E.P. 18520; Herb. R.E.P. 24111; Cult. H.B.C., R.E.P. 19804; Cult. H.B.C., R.E.P. 18944; Herb. R.E.P. 18951; Cult. H.B.C., R.E.P. 18954; Wight 2824; Sebastine 1726; S.coll. 21997; Saulieres 413; Hooper \& Ramaswami 39317; S. coll. acc. no. 474466; S.coll. 17964; Fischer 3163; Herb.Wight 2824; Herb. Wight 2825; Herb. R.E.P. 17518; Cult. H.B.C., R.E.P. 19803; Herb. R.E.P. 17954; Narayanaswami 18887; Blatter \& Hallberg 571; 568; 569; Gamble 
20962; 15605; Perrottet 472; Raghavan 917; 948; 934; 1588; 1592 (all at CAL); Henry 68863; 655; S.coll. 9144; Subramanyam 8278; 5042; Sharma 39952; Narasimhan 1012; Bourne 339; S. coll. 16669; S.coll. 1883; Rathakrishnan 39196; 38066; Subba Rao 36333; Vivekananthan 43078; Vajravelu 41824; 46352; 41822; 41954; 35047; 36905; 44906; 39432; 39487; 38648; 33730; 29273; 21913; 39478; Subba Rao 37437; 40569; Ellis 34842; Sharma, 35581; 36073; Barber 1028; 979; 1041(5); 1041 (2); 1041 (1); 1031; 1029; 1030; Raju 17911; Bridie s.n.; S. coll. 10586; Raju \& Naganathan 4947; 83158; Narayanaswamy 18800; 18883; S. coll. 10175; Herb. H.B.C. 33457; S. coll. 12982; Raju 17816; S. coll. 12071; Daniel \& Raju 86498; Subramanyam 876; Sebastine, 1726; Viswanthan 147; 207; 1098; 598; 1298; 1428; Ramamurthy 17657; Sreemadhavan 388; 856; 838; Ravishankar 95533; Bridie s.n.; Rao 18231 (all at MH); KERALA : Vivekananthan 48620; Herb. Watt. 14984; Cult. H.B.C., Herb. Watt 14978; Anil Kumar 1171; Hook. \& Thomson s.n.; Rama Rao 1730; 1906; Bourdillon 1545; 28; 1258; Meebold 12374; Rottler s.n.; Hohenacker 597; Bourdillon 1391(all at CAL); Nair 67218 (CAL, MH); Ansari 67882; Ansari 67843; Mohanan \& Ramanujam 71993, 71999; Nair 64493; Ramachandran 65291; Ramachandran 63925; Vivekananthan 46666; Ansari 74375; Mohanan 59526; Nair 69831; Vivekananthan 50518; Antony 439; Mohanan 61824; 65187; 56973; 74046; 79235; Ramanujam 72407; Pandurangan 76686; Nair 49885; Ramamurthy 75518 (all at CAL \& MH); Ellis 24059; Antony 767 pp.; Shetty 33507; Vivekananthan 21334; 23972; 21390; 48620; 29322; 50503; Sharma 41641; 42480; Rao \& Raju 8824; Nair 50865; Mohanan 73475; Vajravelu 44703; 26317; Bourdillon 401; Sanbine 210; Anil Kumar 323; 853 (all at MH); KARNATAKA : $S$. coll. 18955; W.A. Talbot 597; Dalzell sn.; Talbot 587; Meebold 10079; Barber 2432, Talbot 2127; Ramesh \& Manohar 8631; Meebold 10078; Banerjee 11714; Ramesh 2320; Akramul Hoque 19903; Wight s.n. (all at CAL); Seshagiri Rao 734351; Seshagiri Rao 73430A; Wadhwa 49222; Singh 133008 ; Raghavan 103877; Ahuja s.n.; Jain 29834; Seshagiri Rao 73654; Ahuja 5946; Das 29708; Singh 141062 ; Raghavan 74095 (all at BSI); Thomson 48; Naithani 21141 (all at MH); GOA : Seshagiri Rao 92957; 84389 (all at CAL); MAHARASHTRA : Duthie 9788; Cult. H.B.C., Burkill \& Prain 16175a; Burkill 31176; Cherian 68213; Puri 6005; Vasavata 5030; 2306 Dhruna 218; (all at CAL); MADHYA PRADESH : G. Watt 14968; Cult. H.B.C., Burkill \& Prain 16180; Cult. H.B.C., Burkill \& Prain 15442; Cult. H.B.C., Burkill \& Prain 15821; Herb. R.E.P. 538; Cult. H.B.C., Burkill \& Prain 15377; Herb. R.E.P.. 35618; Naib Tahsildar, Herb. R.E.P. 35574; Herb. R.E.P. 34930; Narayanaswami 3519; Narayanaswami 3325; Jain 5146; 5238; 5159; Panigrahi \& Singh 4564 (all at CAL); ANDAMAN \& NICOBAR ISLAND : Premanath 8354 (CAL).

Dioscorea pentaphylla L., Sp. Pl. ed.1: 1032.1753; Lam., Encycl. Meth. 3: 234. 1789; Roxb., Hort. Bengal: 72 1814; Roxb., Fl. Ind. (Ed. Carey) 3 : 806. 1832; Wight, Icon. Pl. Ind. Orient. 3(1): 7, t. 814. 1844; Voigt, Hort. Suburb. Calcut. 653. 1845; Kunth, Enum. Pl. 5 : 396. 1850; Thwaites, Enum. Pl. Zeyl. 325. 1864; Prain, J.. Asiat. Soc. Beng., Pt. 2, Nat. Hist. 60: 330. 1891; Hook.f., Fl. Brit. India 6: 289. 1892; Prain, Beng. Pl. 2 : 1066. 1903; T. Cooke, Fl.. Bomb. 3: 264. 1908; Haines, Forest Fl. Chota Nagpur:534. 1910; Prain \& Burkill, J.. Proc. Asiat. Soc. Bengal 10(1): 23. 1914; Merr., Interpr. Herb. Amboin.:: 147. 1917; Duthie, Fl. Gangetic Plain 3(2): 253. 1920; Kunth in Engl., Pflanzenr. 87(IV - 43) : 145. 1924 p.p. (excl. ref. to var. lunata Roth); Haines, Bot. Bihar Orissa 3 : 1173. 1924; C.E.C. Fisch. in Gamble, Fl. Madras 3 : 1056. 1928; Prain \& Burkill, Ann. Roy. Bot. Gard. Calcutta 14(1): 160, t. 57, 66, 67. 1936; Burkill in Steenis, Fl. Males., Ser. 1, Spermat. 4(1) : 315. 1951; H. Ohashi in H. Hara, Fl. E. Himalaya 420. 1966; H. Hara et al., Enum. Fl. Pl. Nepal 1: 68. 1978; Nicolson et al., Interpret. Van Rheede's Hort. Malab.: 292. 1988; Karthik. et al., Fl. Ind. Enum. Monocot.: 75. 1989; Noltie, Fl. Bhutan 3(1) : 10. 1994; Jayas. in Dassan., Revis. Handb. Fl. Ceylon 9: 55. 1995. 
D. pentaphylla var. kussok Prain \& Burkill, J. Proc. Asiat. Soc. Bengal 10(1): 23. 1914; Prain \& Burkill, Ann. Roy. Bot. Gard. Calcutta 14(1): 165. 1936; Noltie, Fl. Bhutan 3(1) : 12. 1994, syn. nov.

Lectotype: Eastern Himalaya, Mungpoo, 2000ft (about 600 m), 9.9.(19)12, P.T. Russell 24, acc. no. 474301 (CAL0000026168!) (designated by Hoque et al., 2018). Syntypes: Eastern Himalaya, Mungpoo, $2500 \mathrm{ft}$ (about $750 \mathrm{~m}$ ), 29.5.1912, P.T. Russell 22, acc. no. 474299 CAL!; Eastern Himalaya, Mungpoo, 3000 ft (bout 900 m), 15.4.1912, P.T. Russell 14, acc. no. 474300 CAL!

Prain and Burkill (1914) discovered another new variety D. pentaphylla L.var. kussok Prain \& Burkill in Mungpo (Sikkim) in the year 1912. They have established their new on the basis of very short, firm, flesh, flavorless tuber with bristles like roots, trifoliolate, reddish glabrescent leaves. All the specimens of $D$. pentaphylla including the type specimens of $D$. pentaphylla var. kussok deposited at CAL have been studied critically to justify the diagnostic values of these cited characters. Notably, D. pentaphylla is with a wide range of distribution around the globe. It has been reported to grow in India to Australia including Nepal, Bhutan, China (S.W.), Japan, Bangladesh, Myanmar, Malaysia, Thailand, Cambodia, Laos, Indonesia, Philippines, Sri-Lanka, Australia, Australia Pacific Islands and New Guinea. After critical examination of all the characters it has been noticed that the features of tubers and the number of leaflets are much variable even in the same individual. Noltie (1994) though mentioned this taxon along with another variety $D$. pentaphylla var. suli Prain \& Burkill and tried to separate both with some obvious characters of leaflets, however, evidently admitted frequent existence of the intermediate specimens (p.188). Therefore, D. pentaphylla var. kussok Prain \& Burkill is considered and recognized as synonym under typical $D$. pentaphylla.

Flowering: July to November; Fruiting: September to January.

Distribution : Usually wild, but cultivated in Malaya and Java. INDIA : Almost all states growing from sea level to an alti-tude of 1900 m; PAKISTAN; NEPAL; BHUTAN; BANGLADESH; MYANMAR; SRI LANKA; CHINA (S.W.); JAPAN; MALAYSIA; THAILAND; CAMBODIA, LAOS; INDONESIA; PHILIPPINES; NEW GUINEA; AUSTRIALIA; AUSTRALIA PACIFIC ISLANDS.

\section{Specimens examined:}

HIMACHAL PRADESH: Johnson s.n. (CAL); UTTAR PRADESH: Mackinnon s.n. (ASSAM); Arora 52418; Herb. R.E.P. 19021, Cult. H.B.C. 14536; Herb. R.E.P. 18034, Cult. H.B.C. 14545; Herb. R.E.P. 18086, Cult. H.B.C. 14510; Herb. R.E.P. 18034, Cult. H.B.C.14520; Kalkaprasad 34846; Panigrahi 2218; Mackinnon s.n.; Mackinnon s.n.; Prasad 1365; King. s.n.; Panigrahi 6542; Bell 774; Gamble 23915; Burkill \& Prain s.n.; Burkill \& Prain s.n.; King s.n.; Herb.R.E.P. 33256; King s.n.; Duthie 7656; King s.n.; Duthie 10578; Kalkaprasad 34804; 34807; Herb. R.E.P. 21101 (all at CAL); Rao 4135; Sarin 3424; Malhotra 23424; Babu 34731; Malhotra 23835; Bhattacharyya 17702; Chawdhery 76290, Singh 338; Murty \& A.K. Goel 1753; Sarin 3336; Dakshini 7272; Malhotra 18092; Malhotra 30710 (all at BSD); SIKKIM: Akramul Hoque 20579; Lister sn.; S.loc. Prain \& Burkill s.n.; King s.n.; King s.n.; Prain \& Burkill s.n.; Prain's Collector s.n.; Akramul Hoque 20580 (CAL), Prain s.n.; Russell 10; Lister s.n. (all at CAL); Hooker s.n. (MH); Raju 6842 (BSHC); WEST BENGAL : Burkill \& Prain s.n.; Paul 507; Bennet 947; Kurz s.n.; Prain s.n. (CAL); Akramul Hoque 18702; Mukherjee 10569; Pal 33336; Akramul Hoque 20503; Herb. R.E.P. 33331; Akramul Hoque 20504; Herb. R.E.P. 35438; Russel 14; Akramul Hoque 20505; Herb.R.E.P. 13915B, Burkill \& Prain s.n.; Herb. R.E.P. 
33450; Akramul Hoque 20506; Narayanaswami \& Party 3070; Pal, Herb R.E.P. 33334; Mukherjee 18751; Pal, Herb R.E.P. 33330; Guha Bakshi 130; Watt. 14555; Haines 700; Watt. 11976, Cult. H.B.C., Prain \& Burkill s.n.; Watt. 1106A, Cult. H.B.C., Prain \& Burkill s.n.; Herb. R.E.P. 13871, Cult. H.B.C., Burkill \& Prain s.n.; Cult. H.B.C., Bourdillon 9; Cult. H.B.C., Lister 3; Das s.n.; Lister s.n., Cult. H.B.C., Prain's Collector s.n.; Prain \& Burkill 7D; Lister s.n.; Banerjee 45; Malick 79; I.H. Burkill 30789; Bhowmik 31; Kurz s.n.; Sengupta 136; Bennet 791; Heawood 31; Chatterjee 18; Lister s.n.; Mann 5; Prain 14539; Cult. H.B.C. 18669.; Herb. R.E.P. 33474; Lister, Herb. R.E.P. s.n., Prain's Cllector s.n.; Janaki Ammal 34/56; Ribu 7; Malick 436; Das 35411; Mukherjee 18721; Roy Chowdhury 32; Burkill \& Prain s.n.; Malick 239; Lister s.n.; Helfer 509; 132; Burkill 24521; Munro 18; Kalkaprasad 35411; Russell 18; Russell 25; Mann 12; Kalkaprasad 19707; Chatterjee 879; Mackinnon s.n.; Ribu, Herb. R.E.P. 34226; Russell 24; Russell 22 (all at CAL); Haines 691 (CAL, MH); Kalika Prashad 33451; Burkill 27532 (all at CAL \& BSIS); Das 34753; Burkill \& Prain 13915B, acc. no. 31237; Kalkaprasad 26581; Burkill 24556; 27367 (all at BSIS); ASSAM: Rao 9861 (CAL, ASSAM); Rao 42425; Deb 31427; Deka 16133 (all at ASSAM); Prain s.n.; Gage s.n.; Bal 363-282; Simons s.n.; Clarke 40729; Shaik Mokim 339; Burkill 32417; Craib 221; Craib 229; Burkill 33041; Craib s.n.; Herb. R.E.P. 3318 (all at CAL); MEGHALAYA: Hooker \& Thomson s.n. (CAL, MH); Hajra 51904; Balakrishnan 46715 (All at CAL \& ASSAM); Balakrishnan 47167; De 19751; Kanjilal 5971 (all at ASSAM); Hooker s.n.; Clarke 45406; Akramul Hoque 20541; 20542; 20550; 20567; 20569; Kapoor \& Party 75401; 75690; Raw 38635; Collett s.n.; Panigrahi 3229; Collett s.n.; Clarke 44487E; Prazer s.n.; Clarke 44688A; Carter 1648; Burkill \& Banerjee 235; 257; Das 370; 35602 (all at CAL); ARUNACHAL PRADESH : Rao 17717; Panigrahi 14721; Rao 20362; Panigrahi 19719 (ASSAM); Panigrahi 14644; Seshagiri Rao 17736; 20301 (all at CAL \& ASSAM ); Akramul Hoque 20513; Burkill 37536; Rao 47943 (all at CAL); NAGALAND : Collett 100; Collett s.n.; Meeebold 7236; Prain s.n. (all at CAL); MIZORAM : Godprey 472; Deb 31427 (All at CAL); MANIPUR : Malick 626; Malick 1178 (all at BSIS); Deb 2648; 587; Meebold 9147 (All at CAL); TRIPURA : Deb 1008; Debbarman 229; 603; 256; 257 (all at CAL); BIHAR : Panigrahi 11711 (ASSAM); Gamble 8739 (MH); Father Cardon 1; 8; Kalkaprasad 19745; 34344; 34346; 34365; 34389; 34390; Father Cardon 11; 12; Clarke 34013A; Cult. H.B.C., Herb.R.E.P. 18490; Das s.n., Herb.R.E.P. 33361; Haines 695; Campbell 9484; Chandra 1355; Burkill \& Prain 13892; Burkill s.n.; Burkill \& Prain 13812; Shetty 340; Herb.R.E.P. 21227 (all at CAL); Rao 22776 (CAL, ASSAM); ORISSA : Rao 29879; Panigrahi 772 (all at ASSAM); Barber 1187 (MH); Carey 95; Watt, Herb. R.E.P. 13922, Prain \& Burkill s.n.; Watt, Herb. R.E.P. 13922, Prain \& Burkill s.n.; Herb. R.E.P. 18210, Cult. H.B.C. 14542; Herb. R.E.P. 21446, Cult. H.B.C. s.n.; Herb. R.E.P. 18615, Cult. H.B.C. 14513; Watt, Herb. R.E.P. 11069, Prain \& Burkill s.n.; Barber 1293, Herb. R.E.P. 20366; Kalkaprasad 34301; Holmes 34309; Kalkaprasad 34323; Holmes 33825; Panigrahi 20975; Watt, Herb. R.E.P. 11069, Prain's Collector s.n.; Watt, Herb. R.E.P. 11069, Prain s.n.; Verma 1570; Mukherjee 4362; Holmes, Herb. R.E.P. 33187; Herb. R.E.P. 33185; Panda. \& A.P.Das.792; Namhata 2544 (all at CAL); ANDHRA PRADESH : Barber 5157 (MH); Barber Herb. R.E.P. 17959, acc. no. 31271; Prain \& Burkill, Herb. R.E.P. s.n.; Prain \& Burkill 11069. acc. no. 31266; Herb. H.B.C. s.n.; Herb. R.E.P. 12683, (all at BSIS); Sebastine 13134; 13114; 6677; Subba Rao 68535; Herb. R.E.P. 14984; Ellis 14943; Subramanyam 6861; 6860 (all at MH \& CAL); Ranga Charyulu 908; Subba Rao 45927; 14947; Ellis 16789; 22173; 32505; 25415; 17988; 22161; Beddome s.n.; Rama Rao 83602; Sebastine 11607; Ravishankar 83173; Subba Rao, 21572; 42590 (all at MH); Herb.R.E.P. 17762, Cult. H.B.C.14563; Herb. R.E.P. 17537; Herb. R.E.P. 35154; Burkill \& Prain 11810A; Prain s.n; Watt, Herb R.E.P. 12683, Prain \& Burkill s.n.; Herb. R.E.P.1810A, 
Burkill \& Prain s.n.; Watt s.n., Herb. R.E.P.12679; Herb. R.E.P. 12682A, Burkill \& Prain s.n.; Herb. R.E.P. 17959, Watt 14541; Prain's Collector 12682; Herb. R.E.P. 17762, Barber 1019; Herb. R.E.P. 12682A, Burkill \& Prain s.n.; Herb.Wight 1871; Mohan 465; Watt s.n., Prain \& Burkill 12683; Gamble 20978 (all at CAL); TAMIL NADU: Narayanaswamy 18991; 3389; Sebastine 1511; 25017; 6978; Henry 285; 289; 530; 531; Ramamurthy 14781; Deb 30885; Jacob 17800; Viswanathan 221; Diwakar 97082; Ragupathy 120; Vajravelu 36707; 41735; 39308; 39494; Shetty 34423; 28025; 32322; Wight 2823; Narasimhan 158; Bidie s.n.; Sreemadhavan 926 (all at MH); Herb. R.E.P. 16953; Herb. R.E.P. 18943, Cult. H.B.C. 14565; Herb. R.E.P. 17753, Cult. H.B.C. 14557; Ramamurthy 47700; Herb. R.E.P. 17752, Cult. H.B.C. 14554; Herb. R.E.P. 35154; Herb. R.E.P. 18541,Cult. H.B.C. 14553; Akramul Hoque, 19905; Hooper \& Ramaswami 39225; Herb. R.E.P. 19799, Cult. H.B.C. 14548; Herb. R.E.P. 19803, Cult. H.B.C. 14560; Hooper \& Ramaswami 39320; Herb. R.E.P. 35150; Ragupathy 120; Sebastine 1511; Perrottet 1; 334; Meebold 13814; Herb. R.E.P. 20842; 20843; Auglade 1864; Ellis 14947; Ragupathy 1164; Balakrishnan 40173; Gamble 14657; Watt. Herb. R.E.P. 11976, Prain \& Burkill s.n.; Bourdillon 1256; Fischer 1245; Herb. R.E.P. 17762, Cult. H.B.C. 14564; Herb. R.E.P., Cult. H.B.C. 26530; Narayanaswami \& Party 331; Kalkaprasad 1; Father Cardon s.n.; Herb. R.E.P. 18945, Cult. H.B.C. 14558 (all at CAL); Viswanathan 1157; 534; Henry 68951; Barber 5387; Subramanyam 1321; 10550; 10551; 1757; 823; 10411; 10410; Ramamurthy 79668; 14794; 79636; 52813; Henry 48265; 48240; 68945; Vivekananthan 46712; Joseph 15168; Sebastine 6974; 1237(all at CAL \& MH); KERALA : Anil Kumar 890; Vivekananthan \& Raju 71127; Mohanan 59592; 56985; 65172; Ramamurthy 48525; 74880; 47680; Ramachandran 57505; 58720; 52247; 62762; Vivekananthan 48668; Chandrabose 49080 (all at CAL \& MH); Vajravelu 26037; Ellis 19973; 20462; 26382; Sebastine 22319; Bhargavan 90094; Joseph 41987; Antony 767 pp.; Vivekananthan 21332; Sharma 42460; Naithani 24664 (MH); Vajravelu 33170; Bourdillon 402; Bourdillon s.n.; Barber 6648; Nair 50867; Sebastine 21089; Ramamurthy 47700 (all at MH); Bourdillon 1388; 1418; 21; 22; 1414; 1264; 1255; Mohanan 79984; Bourdillon, Cult. H.B.C., Prain \& Burkill 7; Herb. R.E.P. 33458; 33459; Herb. Wight 2829; Herb. R.E.P. Hort.Bot.Cal. 33459; Herb. Wight 2823; Calder \& Ramaswami 477; Calder \& Ramaswami 136; 137; 138; Ramaswami 16; Nair 50867; Sukdibaha 33459; Rama Rao. 1428 (all at CAL); KARNATAKA: Naithani 21221; Barber 2262; 2472 (all at MH); Barber 2202 (CAL, MH); Herb. R.E.P. 17039; Keshava Murthy \& Sreenath 2946; Rao 74988; Akramul Hoque 19904; Saldanha \& Ramesh 1769; Talbot 1041; Thomson s.n.; Saldanha \& Prakash 3659; Talbot 799; Saldanha \& Ramesh 2594; Banerjee 11829; Talbot 1978; Saldanha 2223; Herb. R.E.P. 22377, Prain \& Burkill s.n.; Talbot 1042; Herb. R.E.P. 11479, Burkill \& Prain s.n. (all at CAL); GOA: Seshagiri Rao 92933 (CAL); MAHARASHTRA : Billore 116722 (CAL, BSI); BSI,W.C. 115895; Venkata Reddi 99284; Akrmul Hoque 19902; Billore 113101; Puri 13861; Burkill 31337; 31319; Jain 5959; Cherian 65939; Dalzell s.n.; Herb. R.E.P. 17537; Talbot 2622; I.H. Burkill 31180; Hocks s.n.; Herb. R.E.P. 17026; Meebold 8798; Das 34488; Hooper 34608; 34610; Herb. R.E.P. 16041, Cult. H.B.C., Burkill \& Prain s.n. 16041; Dhruna 4; Hooper, Herb. R.E.P. 34475; Dalzell s.n.; Jain 5959 (all at CAL); Raghavan. 103231; Venkata Reddi 93456; Billore 110598; Billore 111870; Jain 8264; Wadhwa 128227; Narasimhan 166030; 166203; Malhotra 144714; S.D.M. 6901; Love 112000; Rao 81448; 66184; Janardhanan 66205; Herb. R.E.P. 13867; Janardhanan 72417; Wadhwa 127661; John Cherian 65939; Janardhanan 72526; Ryan 2346; Ryan 1261; Gammie 15469; Malhotra 151422; Malhotra 144700; Reddi 68343; Torne s.n.; Singh 108973; Malhotra 144443; 144640 (all at BSI); MADHYA PRADASH : Maheshwari 4322; Prain's Collector 14040; Herb. R.E.P. s.n.; Burkill \& Prain 14040; Prain \& Burkill 9157; Herb. R.E.P. 14023, Cult. H.B.C. s.n.; Herb. H.B.C. 35573; Rao 83909; Rao 83895; 
Panigrahi 6542; Narayanaswami 3411; Herb. R.E.P. 33457; Herb. R.E.P. 33458; Jain 5196; Prain \& Burkill 14023; Herb. R.E.P. 20821; Duthie s.n., Herb. R.E.P., Burkill \& Prain s.n.; Burkill \& Prain 15820; Prain \& Burkill 15439; Herb. R.E.P. 33459; Prain \& Burkill 15449; Burkill \& Prain 15467; Herb. R.E.P. 15782, Burkill \& Prain s.n.; Herb. R.E.P. 14050, Burkill \& Prain s.n.; Herb. R.E.P. 15467 (all at CAL); Joseph 11305 pp. (excluding fruit) (MH); Maheshwari 4514; Herb. R.E.P. 24047 (BSIS); RAJASTHAN: Singh 3083 (CAL); Kanodia 75233, (BSI); ANDAMAN \& NICOBAR ISLAND : Helfer 5534; Basu 7361; Nair 6253; Rogers 51; King's Collector s.n.; King's Collector s.n.; King's Collector s.n.; King's Collector s.n.; Prain's Collector 25; Rogers s.n. Herb. R.E.P. 23828; Prain's Collector 9; King's Collector s.n.; King s.n.; King's Collector s.n.; Heinig 268; Prain s.n.; King's Collector s.n.; King's Collector s.n. (all at CAL).

\section{Acknowledgements}

The authors are grateful to the Director, Botanical Survey of India and the Head of office, Central National Herbarium, Botanical Survey of India, Howrah, for giving necessary permission to consult herbarium and library. We are also thankful to Head of the Department, Department of Botany, University of Calcutta for the permission to consult the specimens deposited at CUH. Jayanta Ghosh is warmly thanked for his help.

\section{LITERATURE CITED}

Hoque, A.; Lakshminarasimhan, P. \& Maity, D. 2017. Dioscorea polystachya (Dioscoreaceae): A new record for India. Rheedea 27(2): 152 - 155.

Hoque, A.; Lakshminarasimhan, P. \& Maity, D. 2018a. Dioscorea longipedicellata (Dioscoreaceae), a new species from the Indian subcontinent. Phytotaxa 367(2): $173-178$.

Hoque, A.; Lakshminarasimhan, P. \& Maity, D. 2018b. Lectotypification of thirteen names in Dioscoreaceae R.Br. Pleione 12(2): 299 - 308.

Hoque, A. \& Mukherjee, P.K. 2002. A new name for Dioscorea glabra var. hastifolia Prain et Burkill from the Andaman and Nicobar Islands, India. J. Bomb. Nat. Hist. Soc. 99(2): $371-373$.

https://wcsp.science.kew.org/qsearch.do

Jayasuriya, A.H.M. 1995. Dioscoreaceae. In: Dassanayake, M.D. (ed.), A Revised Handbook to The Flora of Ceylon Vol. 9. Amerind Publishing Co. Pvt. Ltd., New Delhi. Pp. 47 - 80.

Karthikeyan, S.; Jain, S.K.; Nayar, M.P. \& Sanjappa, M. 1989. Dioscoreaceae. In: Florae Indicae Enumeratio: Monocotyledonae. Botanical Survey of India, Calcutta. Pp. $73-76$.

Mabberley, D.J. 2017. Dioscoreaceae. In: Mabberley's Plant-Book, A Portable Dictionary of Plants, Their Classification and Uses. $4^{\text {th }}$ Edn., Cambridge University Press, Cambridge. Pp. 298.

Noltie, H.J. 1994. Flora of Bhutan. Vol. 3, part 1. Royal Botanic Garden Edinburgh, Edinburgh. Ppl 4 - 17.

Prain, D. \& Burkill, I.H. 1914. A synopsis of the Dioscoreas of the Old World, Africa excluded, with descriptions of new species and varieties. J. \& Proc. Asiat. Soc. Bengal 10(1): 5-41. 